EUROPEAN ORGANIZATION FOR NUCLEAR RESEARCH

CERN SL/2000-042 AP

CERN-NUFACT Note 32

\title{
R\&D Needs for a Neutrino Factory
}

\author{
E. Keil
}

\begin{abstract}
The R\&D needs for a neutrino factory, based on a muon storage ring, are presented. Such a neutrino factory consists of several modules. All these modules must be developed, namely: Intense proton source, pion production target and collection system, ionization cooling, acceleration and storage of the muons. R\&D on these modules will be discussed in terms of theory and simulation, engineering prototypes, and experiments in particle beams.
\end{abstract}

Invited paper at NuFact'00, Monterey CA, USA, 22 to 26 May 2000

Geneva, Switzerland

July 31, 2000 


\section{WHY DO WE NEED RESEARCH AND DEVELOPMENT (R\&D)?}

Figure 1 shows a schematic layout of a neutrino factory (NF) [1]. Its design uses novel concepts for several different modules, e.g. proton sources, proton targetting, $\pi / \mu$ collection, $\mu$ cooling, $\mu$ acceleration, and $\mu$ storage. The novelty reaches a peak in the middle of the list. It follows that R\&D on NF must be done on many fronts at the same time. This is in contrast to linear $\mathrm{e}^{+} \mathrm{e}^{-}$colliders LC [2] and very large hadron colliders VLHC [3] that use novel concepts on only a few types of modules that are repeated many times, e.g. accelerating sections, wave guides and RF power sources for LC, arc magnets for VLHC, and tunnel construction techniques for both. If we could work out a conceptual design for a NF of adequate performance without R\&D we would do it.

While doing R\&D it is important to keep in mind that its goal is advancing the understanding in order to be ready for a Conceptual Design Report in a few years. Such R\&D ought to be done in cycles that last about a year each and reconcile two conflicting aims, consistency and openness for better ideas. Consistency means that the beam parameters at the module boundaries agree, preferably more than less. In each cycle, one should define a scenario [1], i.e. a set of parameters for the beam, modules and performance of a neutrino factory which has module parameters that are either within reach now or will be in future. The scenario also orients the engineering of prototypes. Arranging R\&D in cycles permits replacing modules and/or scenarios by better ones between cycles. The recent NF study at Fermilab [4] is an example of such an R\&D cycle.

R\&D for a NF may be discussed in terms of three classes that I call R\&D proper, prototyping and experiments. R\&D proper comprises analytical calculations and simulations that lead to a scenario. It consists of the following detailed steps: (i) Develop and verify theory (ii) Find or develop and verify simulation tools (iii) Find and optimize module parameters, using theory, simulation, and advice from applied physicists and engineers (iv) Optimize NF by shifting module boundaries. Examples are: 1. Balancing the cost reduction from less cooling, leading to larger emittances in all downstream modules, and the cost increase from the larger apertures there. 2. Increasing e.g. the output energy of the linear accelerator reduces the aperture needed in the downstream modules, and may allow a more efficient packaging of the RF cavities in the recirculating linear accelerators, and vice versa. Such optimizations need cost figures for at least one NF, and scaling laws for the cost variation with the module parameters. Finding them is an R\&D activity.

Prototyping is an activity of applied physicists and engineers who develop prototypes for the components of NF modules. In detail, it consists of the following typical steps: conceive, engineer, build and test.

Finally, experiments are the testing of NF components in a beam. Such experiments are very motivating for the NF team, since they oblige them to assemble a system of modules that fit together, and to perform the experiment within the boundaries of a machine schedule. They may be needed to convince funding agencies that a NF is 
worth funding.

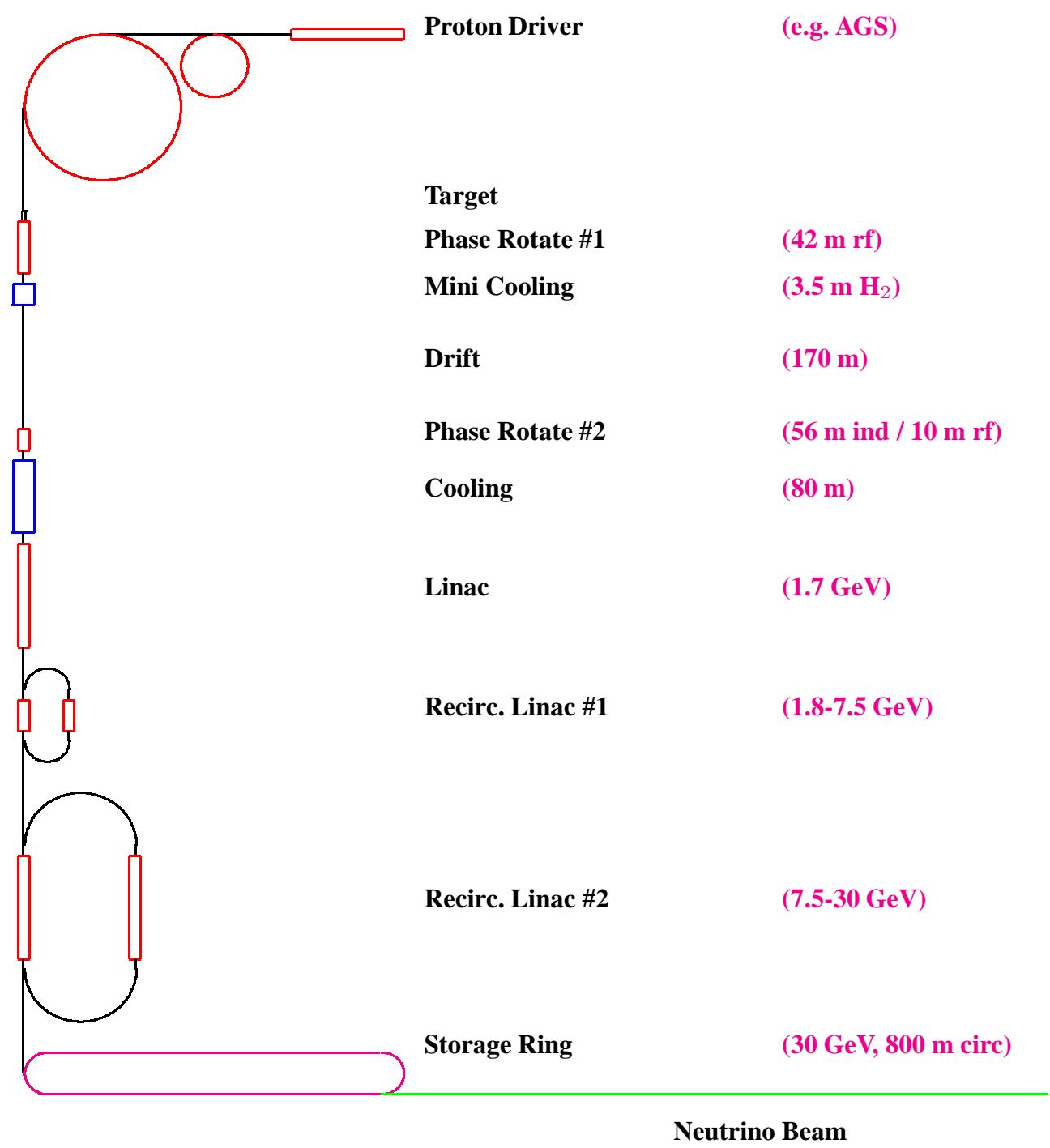

Figure 1: Generic neutrino factory layout [1] showing the most important modules. The proton driver consists of rapid-cycling synchrotrons in cascade, and puts a MW proton beam onto the target. The phase rotations and the drift space between them use the correlation between longitudinal $\mu$ momentum and velocity to reduce the energy spread in the $\mu$ beam. Cooling reduces the normalized emittance by about an order of magnitude in each transverse plane. Linac and two recirculating linacs accelerate the $\mu$ beam that is stored in the storage ring.

Of course, splitting R\&D into three classes is a simplification. Many problems fall into more than one class. All the time, one should use simulation to verify theory and vice versa, use insight into future engineering possibilities, and choose parameters 
in ranges that might be achieved in practice. All this needs mutual understanding between theoretical accelerator physicists, applied physicists and engineers about their respective problems.

\section{R\&D ON NEUTRINO FACTORY COMPONENTS}

This chapter contains a discussion of the R\&D needs of five NF modules, namely proton sources, targetting and capture, cooling, acceleration and storage.

\subsection{R\&D on the proton source}

The proton source should deliver a few short high-intensity bunches with a few MW of beam power $W$. This requirement is different from that in spallation neutron sources that also deliver a few MW of beam power, but in many long low-intensity bunches. The proton losses should be small in order to permit hands-on maintenance, and to avoid remote handling over most of the length of the proton source. According to Monte Carlo codes, simulating particle production, the number of $\pi / \mu$ from a target is proportional to $W$, but relatively insensitive to the proton energy $2 \leq E \leq 30 \mathrm{GeV}$. It follows that the proton flux is $N \propto 1 / E$.

The proposed proton sources are inspired by existing synchrotrons and spallation neutron sources, and rely heavily on equipment that is or will be available at various sites for reasons unrelated to NF. A super-conducting linear accelerator $[5,6,7]$ with circular pulse accumulator and compressor [8] is being studied at CERN. Rapidcycling synchrotron(s) are favoured elsewhere. Lower energy synchrotrons can cycle faster. If one assumes that the product $f_{\text {rep }} E$ of repetition frequency $f_{\text {rep }}$ and proton energy $E$ and the proton beam power $W$ are constant, then one can show that all synchrotrons have equal numbers of protons $N$ in a cycle.

All synchrotrons must accelerate large numbers of protons $N$ in a cycle, with injection at relatively low energy. This implies their performance is limited by ordinary space charge and other collective effects. All these effects need further study.

\subsection{R\&D on Targetting and Capture}

Figure 2 shows the schematic layout of targetting and capture [1]. The R\&D on targetting and capture concentrates on the following issues:

- Target material: Solid graphite - liquid Hg jet - levitated moving band

- Magneto-hydrodynamics of $\mathrm{Hg}$ jets, moving conductors in a magnetic field

- Field level - lifetime - radiation - heating - stresses - shielding of the resistive solenoid surrounded by a super-conducting solenoid

- Choice between solenoid channel and magnetic horns 


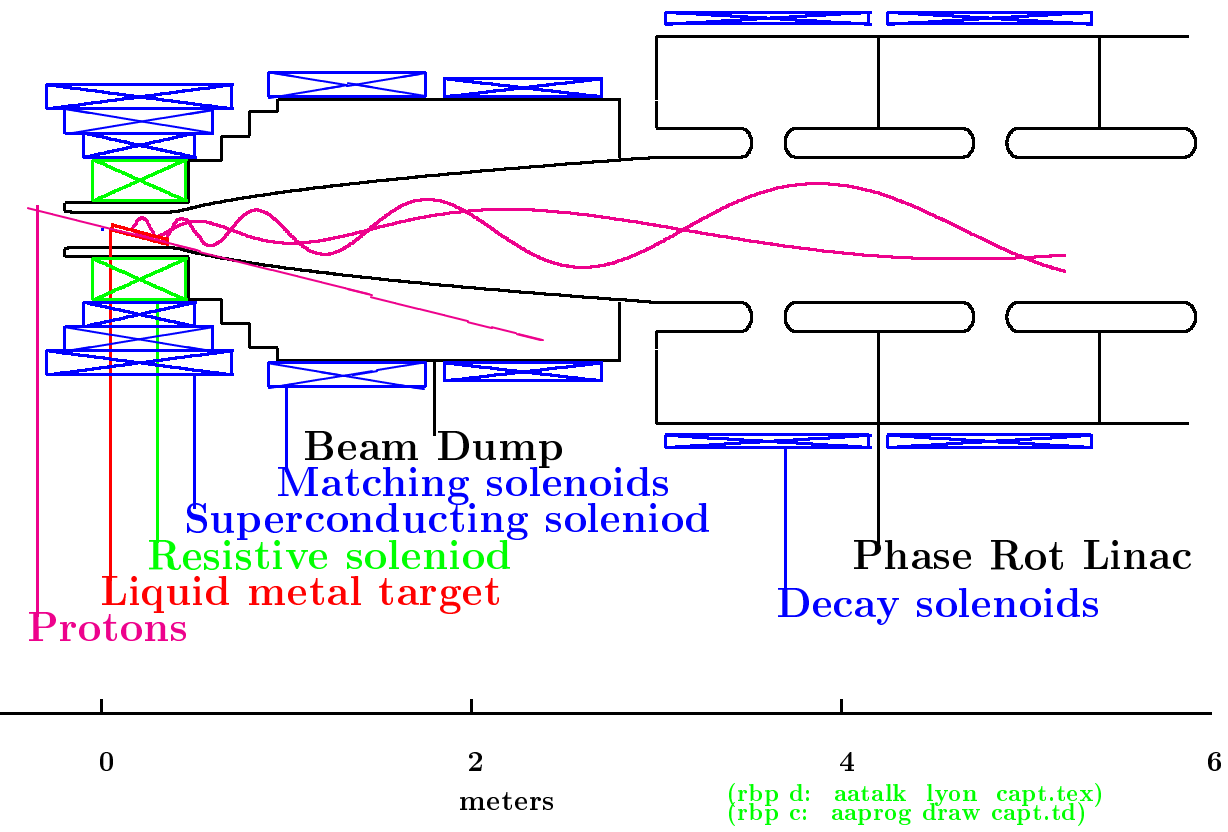

Figure 2: Targetting and capture. The protons enter from the left and strike a liquid $\mathrm{Hg}$ jet that has an angle with the beam axis. The target is inside a $20 \mathrm{~T}$ magnetic field, generated by a resistive solenoid surrounded by a super-conducting solenoid. Tapered matching solenoids match the $\pi$ and $\mu$ beam into the $1.25 \mathrm{~T}$ decay solenoids. The energy spread in the beam is reduced by "phase rotation" in an RF system which uses the correlation between $\mu$ energy $E$ and their velocity $\beta$ to accelerate the low energy, and to decelerate the high energy $\mu$.

- Choice between induction linac in Fermilab study [4] and RF systems in CERN study for "phase rotation" [9]

The targetry experiment E951 [10] is an approved experiment at BNL, coordinated by K.McDonald of Princeton U. Its goals are to demonstrate performance and lifetime of solid and liquid $1 \mathrm{MW}$ targets in a high-field solenoid, to measure particle yields, and to compare them to Monte Carlo codes. The E951 experiment will include the following steps over the next few years:

- Complete beam line A3 at BNL

- Assess mechanical behaviour of target by thermal calculations

- Develop 20 T solenoid and 70 MHz high-gradient RF cavity

- Test solid target in beam

- Test liquid Hg jet in high magnetic field at NHMFL in Florida

- Complete tests with beam at $10^{14} \mathrm{p} / \mathrm{pulse}$ 
Similar, but hopefully complementary target tests may be carried out in Europe. The approved experiment HARP (PS214) at CERN [11] will obtain particle production data for proton energies between 2 and $15 \mathrm{GeV}$ in 2001 .

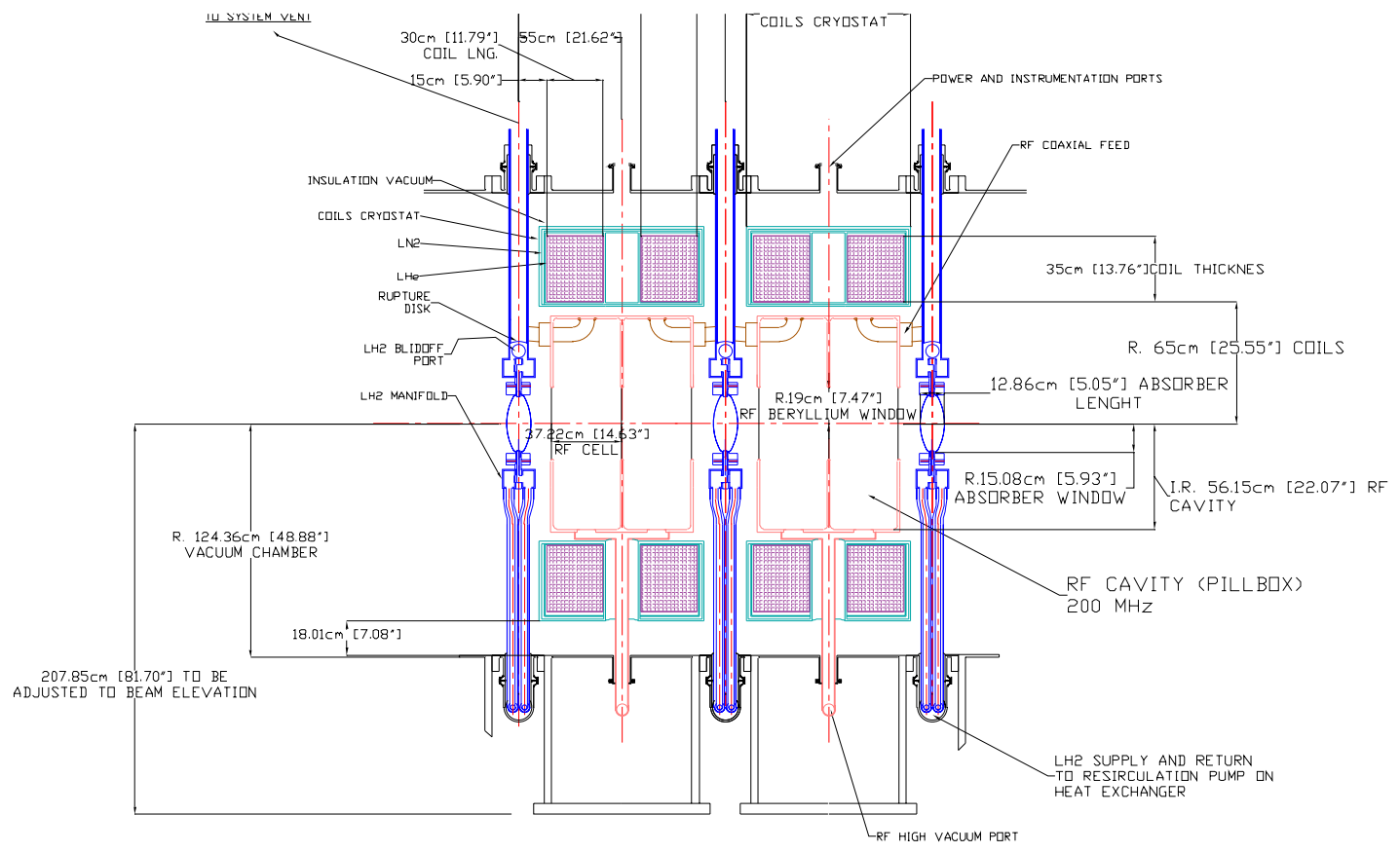

Figure 3: Engineering drawing [4] of two sections or one lattice period of the FOFO cooling lattice, showing two pill-box type RF cavities with a Be window, three liquid $\mathrm{H}_{2}$ absorbers, four solenoid coils, and many connections.

\subsection{R\&D on Muon Cooling}

The equation for ionization cooling of the normalised transverse emittance $\varepsilon_{n}$ [12]

$$
\frac{\mathrm{d} \varepsilon_{n}}{\mathrm{~d} s}=-\frac{\varepsilon_{n}}{\beta^{2} E} \frac{\mathrm{d} E}{\mathrm{~d} s}+\frac{\beta_{\perp} E_{s}^{2}}{2 \beta^{3} m_{\mu} c^{2} L_{r} E}
$$

contains the negative cooling term and the positive heating term with the characteristic scattering energy $E_{s} \approx 13.6 \mathrm{MeV}$. The heating term is kept small if the optics of the cooling channel is arranged such that the transverse betatron amplitude function $\beta_{\perp}$ is small, and the radiation length $L_{r}$ is large. Cooling and heating rates are both inversely proportional to the muon energy $E$. Figure 3 shows a typical cooling cell [4]. The ionization loss in the liquid $\mathrm{H}_{2}$ absorber with $\mathrm{Al}$ or Al-Be alloy windows is about $4 \mathrm{MeV}$. A typical $\mu$ beam deposits about $100 \mathrm{~W}$ in the absorber. The fluid dynamics and thermal modelling of the absorber are a challenge. A high-gradient RF 
system compensates the ionization loss. The RF cavities have Be windows or grids of Al tubes across the beam aperture, in order to achieve a high enough shunt impedance. Solenoid focusing surrounds absorber and RF cavities to achieve a small $\beta_{\perp}$ at the absorber.

The $\mu$ scattering experiment MUSCAT [13] at TRIUMF by a Birmingham, IC, RAL, Riken, UCLA collaboration aims at measuring scattering angles accurately, and thus distinguishing between various alternative theories of multiple scattering.

An ambitious $\mu$ cooling experiment MUCOOL [14] at Fermilab was originally proposed to demonstrate cooling for a $\mu^{+} \mu^{-}$collider. Its adaptation to a NF is under way. Any cooling experiment will be difficult. The tracking devices must measure accurately the expected emittance reduction that is a few percent, RMS scattering angles that are of the order of a mrad, and straggling that is of the order of an MeV. In order to achieve these goals, the mass in the tracking devices must be very small.

Everybody I know and myself believe that ionization cooling will eventually work. However, since MUCOOL was once proposed, presumably because it was considered necessary and/or useful at the time, some cooling experiment or demonstration is essential for a NF. Its failure would be a severe blow to a NF. Apart from serving as a basis for the design of the cooling section in the real NF, such an experiment will demonstrate the beam diagnostics needed for setting up the real NF, not only the cooling experiment proper, and provide a focus for the design of its components.

\subsection{R\&D on Muon Acceleration}

A linear accelerator and typically two recirculation linear accelerators (RLA) similar to CEBAF [15] accelerate the muons. The muon energies at the output end of the linear accelerator and the first recirculating linear accelerator can be found by a cost optimization, varying RF frequencies, normalized transverse emittance, energy spread and the lattice of the RLA. Fig. 4 shows the optical parameters in the first RLA at CERN. It has the shape of a racetrack with linear accelerators in both long straight sections. The number of muon passes is four. The lattice of the linear accelerators is a simple FODO lattice with one RF module in every half cell. The focusing is adjusted such that the betatron wavelength, and hence the $\beta$-functions, are constant on the first pass. In the subsequent passes, the focusing is weaker because of the higher energy, and the $\beta$-functions are larger and vary along the linear accelerators. The arcs consist of isochronous pairs of double-bend achromats. The spreaders and combiners that feed the beams from the linear accelerators into the separate arcs, and vice versa, are not yet designed, and replaced by dummy matching sections for the $\alpha$ and $\beta$ functions.

The total accelerating RF voltage in the two recirculating linear accelerators RLA is more than $12 \mathrm{GV}$ at CERN, and about $11 \mathrm{GV}$ in the Fermilab study in which acceleration was identified as one of the cost drivers [4]. Super-conducting RF is the only way of avoiding a peak RF power that is far too large. R\&D towards higher gradients is desirable, because they make the RLA circumference smaller, reduce decay losses and beam loading, but also need shorter bunch trains. Efficient single-turn injection 


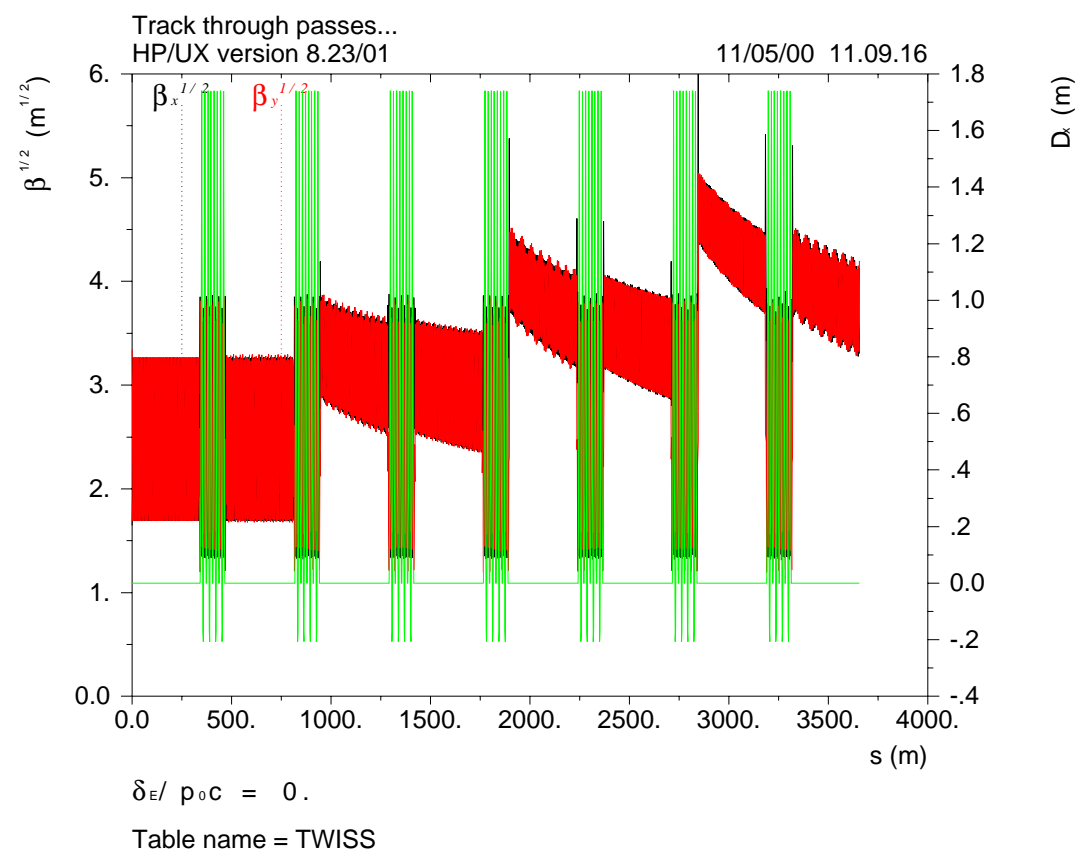

Figure 4: Optical Functions in the four passes through $\mu$ RLA1 at CERN. The black and red (dark grey in the printed version) curves show horizontal and vertical amplitude functions $\sqrt{\beta_{x}}$ and $\sqrt{\beta_{y}}$, respectively. The green (light grey) curve is the horizontal dispersion $D_{x}$.

requires that the bunch trains are shorter than the circumference of the smaller RLA. The beam loading at the muon fluences shown in Table 1 and repetition rates of few tens of $\mathrm{Hz}$ is severe. At CERN, isochronous RLA accelerate the $\mu$ on the crest of the RF waveform, while at Fermilab an-isochronous RLA accelerate the $\mu$ off the crest of the RF waveform. The energies at which the $\mu$ are transferred from the linear accelerator into $\mu \mathrm{RLA} 1$ and from $\mu \mathrm{RLA} 1$ into $\mu \mathrm{RLA} 2$ remain to be optimized, taking into account two facts. A larger normalised emittance and/or a lower injection energy imply a lower RF frequency, because of the scaling of the beam apertures of the RF cavities with frequency. A larger initial relative energy spread implies fewer passes in RLA, since the energy spread must be much smaller than the ratio of the energies between passes.

\subsection{R\&D on Muon Storage Rings $(\mu \mathrm{SR})$}

Tab. 1 shows a comparison of the muon storage ring parameters at Fermilab [4] and CERN [16]. The CERN design aims for 2.8 times the $\nu$ flux/s of the Fermilab design. Hence, it is more demanding than the Fermilab design on proton source, targetting, 
collection, cooling, and shielding. However, the CERN design is less demanding on emittance $\epsilon_{\mathrm{xn}}$, momentum spread $\sigma_{e}$, physical and dynamic aperture for acceleration and storage.

Table 1: Comparison of the muon storage ring parameters at Fermilab and CERN

\begin{tabular}{lrrl}
\hline & Fermilab & CERN & \\
\hline Energy & 50 & 50 & $\mathrm{GeV}$ \\
Shape & Racetrack & Triangle & \\
Distance to detector(s) & $\approx 3000$ & $1000 \& 3000$ & $\mathrm{~km}$ \\
Year & $2 \cdot 10^{7}$ & $10^{7}$ & $\mathrm{~s}$ \\
Muon fluence/detector & $2 \cdot 10^{20}$ & $2.8 \cdot 10^{20}$ & $1 / \mathrm{y}$ \\
Normalised emittance $\epsilon_{\mathrm{xn}}$ & 3.2 & 1.67 & $\mathrm{~mm}$ \\
Relative RMS energy spread $\sigma_{e}$ & 1.0 & 0.5 & $\%$ \\
Circumference & 1.753 & 2.075 & $\mathrm{~km}$ \\
\hline
\end{tabular}

In the triangular CERN design, three long straight sections are connected by three arcs, as schematically shown in the mimic diagram at the top of Fig. 5. The first two long straight sections point at two remote detectors at 1000 and $3000 \mathrm{~km}$. The third long straight section is used for fine adjustments of the tunes. The arcs are composed of rather compact FODO cells. Between the arcs and the long straight sections are dispersion suppressors with modified bending, and matching insertions for the $\alpha$ and $\beta$ functions. The Fermilab design has the shape of a racetrack with two long straight sections joined by two arcs. One of them points at a single remote detector at about $3000 \mathrm{~km}$. The weak focusing and the associated large $\beta$-functions in the first long straight section(s) of both designs achieve the required value of the normalized divergence $\sigma^{\prime} \gamma \approx 0.1$.

In both designs, the first cycle of optical work is essentially done with few outstanding items, in particular a study of the effects of alignment and field shape errors, and their correction, an improvement of the chromatic behaviour, and further studies of the consequences of fringe fields. Obtaining the dynamic aperture by tracking realistic distributions of more than $10^{4}$ muons through acceleration and storage ring for the full muon life time is easy.

The Fermilab study includes a fair amount of engineering, the CERN study practically none. Future R\&D on $\mu$ SR should include engineering studies of the packaging, installation and operation of the components in tunnels with the slopes required for the long baselines pointing at the $\nu$ detectors. The engineers should propose changes to engineering parameters, e.g. magnetic fields $B$, etc., that make a NF easier to build and that reduce its cost. The accelerator physicists should reconsider values for the $\mu$ beam parameters normalised emittance $\varepsilon_{n}$, relative momentum spread $\delta$, muon fluence $\dot{N}$, and their relation to the module parameters. Only afterwards, another round of optical studies, using results of engineering and optimization, should be launched. This task is facilitated by the automated generation of data with Mathematica procedures [17] that 


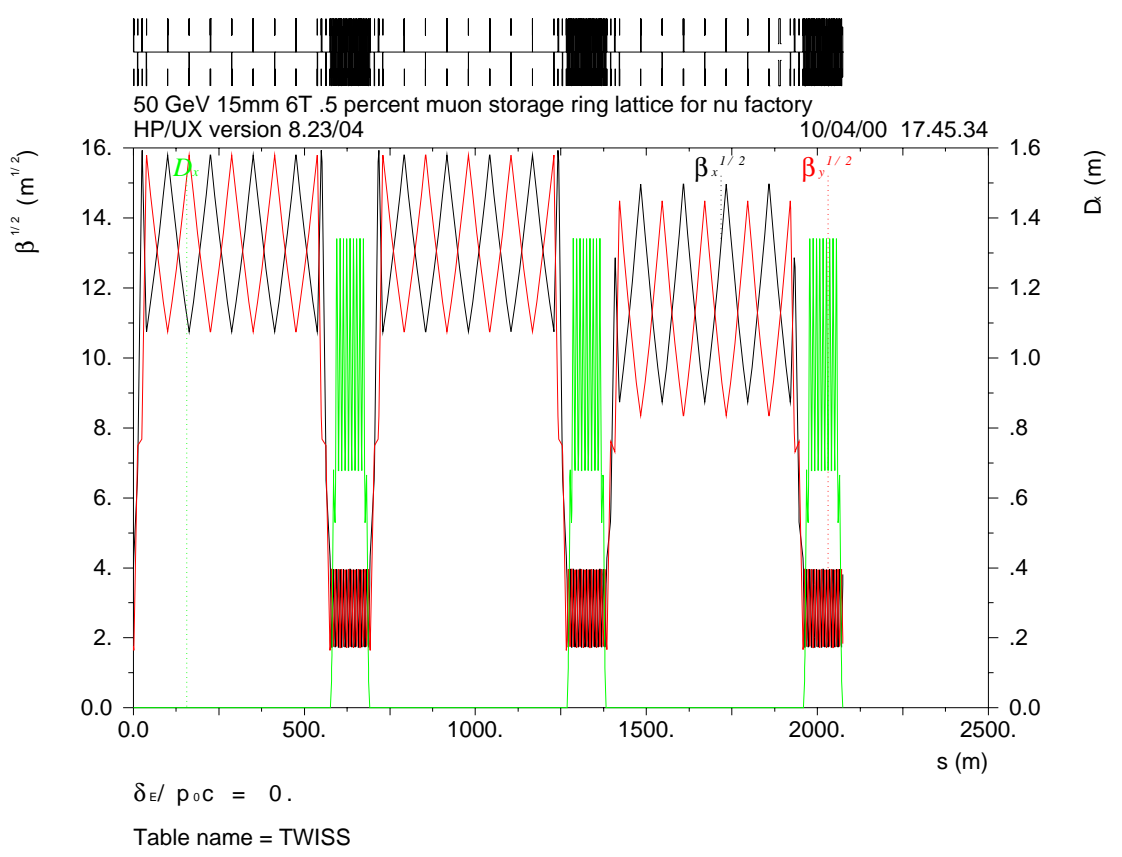

Figure 5: Optical Functions of the triangular $\mu$ SR at CERN. The mimic diagram at the top shows the layout. The three open structures with horizontally focusing (up) and horizontally defocusing quadrupoles (down) are the $500 \mathrm{~m}$ long straight sections. The black rectangles are the three arcs. On either side of the arcs are matching sections. The graph shows amplitude functions $\sqrt{\beta_{x}}$ in black, $\sqrt{\beta_{y}}$ in red or dark grey, and the horizontal dispersion $D_{x}$ in green or light grey.

guarantee the correct geometry, get the thin-element strengths for most optical modules, and feed these data into an optical program such as MAD [18] for thick-element matching, tracking, etc.

\section{CONCLUSIONS}

In the discussion of future directions for NF R\&D I assume that a proof of the feasibility will be achieved soon, mostly by theory and simulation, but also by some prototyping and experimentation. This will allow us to put less emphasis on the internal optimization of the modules, and more emphasis on optimization across modules, by shifting module boundaries, varying the muon energy, and including the cost of the detector(s). Before NuFact'00, I had understood that the figure of merit of an NF was simply the product $E I M$ of energy $E$, fluence $I$ and fiducial detector mass $M$, and that the goal of an overall optimization, including the detector(s), was achieving the maximum possible value of $E I M$, perhaps within a given cost figure. After NuFact'00, I 
understand that two separate figures of merit, $E$ and $I M$, should be considered. In order to have an operating NF sooner and/or at a lower price, one might consider staging its construction, making steps in muon fluence $I$, e.g. by steps in proton beam power and in muon cooling, and in muon energy $E$. Because of the large number and variety of topics, R\&D for an NF offers a wide scope for collaboration on a global scale.

\section{References}

[1] R.B. Palmer, C. Johnson and E. Keil, CERN SL/99-070 (AP) (1990)

[2] http://www.slac.stanford.edu/xorg/ilc-trc/Table1_1.html

[3] http://vlhc.org

[4] N. Holtkamp and D. Finley (eds.), A Feasibility Study of a Neutrino Source Based on a Muon Storage Ring, FERMILAB-PUB-00-108-E (2000)

[5] R. Garoby et al., Feasibility Study of a $2 \mathrm{GeV}$ Super-Conducting $\mathrm{H}^{-}$Linac as Injector for the CERN PS, Proc. XIX Internat. Linac Conf. (Chicago 1998) 106, also CERN/PS 98-049 (RF/HP)

[6] D. Boussard et al., Report of the study group on a super-conducting proton linac as a PS injector, CERN-PS-98-063 (1998)

[7] A. Lombardi and M. Vretenar (eds.), Conceptual Design of a $2 \mathrm{GeV}-4 \mathrm{MW}$ Superconducting $\mathrm{H}^{-}$Linac at CERN, CERN Yellow Report to be published.

[8] R. Cappi et al., Design of a $2 \mathrm{GeV}$ Accumulator-Compressor for a Neutrino Factory, Poster THP1B07 at EPAC2000 (Vienna, Austria, 2000)

[9] A. Lombardi, A 40-80 MHz system for phase rotation and cooling, these proceedings

[10] K. McDonald, Targetry Experiment at BNL, these proceedings

[11] M.G. Catanesi et al., Proposal to study hadron production for the neutrino factory and for the atmospheric neutrino flux, CERN-SPSC/99-35 (1999)

[12] C.M. Ankenbrandt et al., Status of muon collider research and development and future plans, Phys.Rev. ST Accel.Beams 2 (1999) 081001 and references therein

[13] R. Edgecock, Muon Scattering Experiment, these proceedings

[14] C.M. Ankenbrandt et al., Ionization Cooling Research and Development for a High Luminosity Muon Collider (Proposal P904, Fermilab 1998)

[15] Ch. Reece, Overview of CEBAF Operations and SRF-Related Activities at Jefferson Lab, JLAB-ACT-99-08, 9th SRF Workshop, Santa Fe NM, 1-5 Nov 1999.

[16] E. Keil, A 50 GeV Muon Storage Ring Design, CERN SL/2000-013 AP (2000)

[17] E. Keil, Muon Storage Ring Design with Simple Mathematica Packages, CERN SL/99-053 AP (1999)

[18] H. Grote and F.C. Iselin, CERN SL/90-13 (AP) Rev.4 (1995). 